\title{
Les jumelages internationaux des villes de la mer Noire : dynamiques spatiales et temporelles
}

International Town-twinnings of the Black Sea Cities, Spatial and Temporal

Dynamics

Die Städtepartnerschaften in der Schwarzmeerregion: zeitliche und räumliche

Dynamiken

\section{Emmanuelle Boulineau}

\section{OpenEdition}

\section{Journals}

Electronic version

URL: http://journals.openedition.org/rge/5814

ISSN: $2108-6478$

\section{Publisher}

Association des géographes de l'Est

Printed version

Date of publication: 1 December 2016

ISSN: 0035-3213

\section{Electronic reference}

Emmanuelle Boulineau, «Les jumelages internationaux des villes de la mer Noire : dynamiques spatiales et temporelles », Revue Géographique de l'Est [Online], vol. 56 / n³-4 | 2016, Online since 06 December 2016, connection on 08 September 2020. URL : http://journals.openedition.org/rge/5814

This text was automatically generated on 8 September 2020

Tous droits réservés 


\section{Les jumelages internationaux des villes de la mer Noire : dynamiques spatiales et temporelles}

International Town-twinnings of the Black Sea Cities, Spatial and Temporal

Dynamics

Die Städtepartnerschaften in der Schwarzmeerregion: zeitliche und räumliche

Dynamiken

Emmanuelle Boulineau

\section{Introduction}

1 Située entre l'Europe, l'Asie et le Proche-Orient, la mer Noire constitue un laboratoire pour étudier les dynamiques des espaces dans la mondialisation. De nombreuses études ont montré l'intérêt de cette région et sa place dans la structuration des flux mondiaux. La mer Noire est le lieu d'intenses flux d'hydrocarbures et ses hinterlands s'élargissent grâce aux nouveaux terminaux d'hydrocarbures et à l'aménagement de grandes infrastructures.

2 D'un point de vue géopolitique, l'espace de la mer Noire a connu des recompositions majeures. Dans cette zone, trois pays directement frontaliers de la mer Noire sont nés de la disparition de l'URSS en 1991; l'adhésion de la Bulgarie et de la Roumanie à l'Union européenne (UE) en 2007 a contribué à intégrer la rive occidentale dans les dynamiques européennes. Mais les Etats bordiers de la mer Noire sont placés dans l'orbite des grands centres économiques et géopolitiques mondiaux qui se structurent et s'affrontent aussi dans cette partie du monde : l'Union européenne a atteint les rives de la mer Noire, l'adhésion de la Turquie n'y est pas encore acquise, et la Russie guide l'Union économique eurasiatique. Cette position d'entre-deux a de nombreuses conséquences internes à chaque Etat. Elle se manifeste notamment par des 
sécessionnismes régionaux, dans le Caucase et en Moldavie, et par le conflit qui déchire l'Ukraine actuellement et pèse, de ce fait, sur les dynamiques de coopération.

3 Cette fragmentation de l'espace régional autour de la mer Noire s'accompagne en parallèle d'une inscription dans la mondialisation au travers de nombreux accords et traités guidés par les Etats et les grandes institutions internationales : on parle alors de régionalisme (Beckouche, 2013). La multiplication de ces traités est impressionnante: Coopération économique de la mer Noire (mieux connue sous son acronyme en anglais de BSEC, 1992), Commission mer Noire (1992), GUAM (organisation pour la démocratie et le développement regroupant actuellement la Géorgie, l'Ukraine, l'Arménie et la Moldavie, 1997), Forum de la mer Noire (2006) etc... L'Union européenne joue un rôle dans la région au travers de politiques et d'accords: INOGATE (1995) concernant la coopération énergétique, TRACECA (1998) concernant le transport, synergie de la mer Noire (2007) et partenariat oriental (2009) dans la cadre de la politique européenne de voisinage. La structuration d'une région autour de la mer Noire et son insertion dans les flux mondiaux se construit progressivement à l'initiative des principaux acteurs géopolitiques d'ordre étatique ou international, mais elle se heurte aux fragmentations territoriales soulignées plus haut (Sieca-Kozlowski et Toumarkine, 2000 ; Drevet, 2010 ; Serebrian, 2010).

4 Dans ce double mouvement de fragmentation versus coopération dans un espace en voie de mondialisation, cet article propose d'entrer par un autre champ de la mondialisation, celui de la coopération territoriale entre les villes comme vecteur de leur internationalisation. Au travers de l'analyse des jumelages de villes, il étudie la structuration de l'espace de la mer Noire en une région connectée au reste du monde. Il sort ainsi de la perspective déjà bien documentée dans la littérature scientifique des coopérations aux échelles nationales et internationales pour explorer comment les villes, ces nouveaux acteurs de l'internationalisation, construisent à bas bruit des réseaux de coopération territoriale. Il postule que ce type de coopération fondée sur des liens faibles, par opposition aux liens forts des relations géopolitiques guidés par les États, contribuent à la compréhension des dynamiques spatiales dans les processus de construction régionale dans un monde globalisé (Boulineau, 2016).

5 L'internationalisation des villes prend plusieurs formes. La compétition entre les villes dans le cadre de la mondialisation en est une, elle ne sera pas abordée ici. La coopération territoriale en est une autre : elle vise à construire des liens de partenariat internationaux entre les villes, à l'initiative de ces dernières. La reconnaissance des villes comme acteurs autonomes est inscrite dans la charte de l'autonomie locale du Conseil de l'Europe entrée en vigueur dans tous les pays frontaliers de la mer Noire : La Turquie en 1993, la Bulgarie en 1995, la Roumanie, la Russie et l'Ukraine en 1998, la Géorgie en 2007. Elle a été aussi engagée, à divers degrés selon ces pays, dans les politiques de décentralisation. Ces formes d'internationalisation ne sont pas exclusives les unes des autres. Les jumelages montrent que la dimension stratégique de l'insertion dans la compétition entre les villes s'articule avec l'échange d'expériences au sein d'un partenariat (Clarke, 2009 ; Joenniemi et Sergunin, 2011).

6

Dans les six pays bordiers de la mer Noire que nous avons étudiés (Bulgarie, Géorgie, Roumanie, Russie, Turquie et Ukraine), l'ouverture internationale a débuté avec celle des Etats : décennie 1980 pour la Turquie, ruptures de 1989 pour la Bulgarie et la Roumanie et de 1991 pour l'espace post-soviétique. Des villes très importantes comme Istanbul (environ 13 millions d'habitants) possèdent depuis longtemps un rayonnement 
international. D'autres villes millionnaires comme Rostov-sur-le-Don et Odessa dominent l'espace régional. Par ailleurs, de nombreuses villes de la mer Noire sont des ports, ce qui facilite les contacts internationaux depuis longtemps. Nous étudierons ainsi comment la dynamique temporelle et spatiale des jumelages de villes reflète ou non ce mouvement d'ouverture internationale et de construction d'une région mer Noire, en nous appuyant sur les résultats du programme de recherche européen ESPON ITAN auquel nous avons participé ${ }^{1}$.

\section{Etudier les jumelages de villes : définitions et méthodes}

\section{A. Approches de l'étude des jumelages de villes}

7 La bibliographie sur les jumelages de villes est réduite si on la compare à celle qui concerne l'internationalisation des villes et leur mise en compétition. Mais dans les deux cas, cette littérature est très marquée par une lecture européenne voire occidentale des jumelages et de l'internationalisation des villes car la bibliographie que nous avons consultée est de langue anglaise ou française : elle prend donc un point de vue européano-centré ou elle se place dans le paradigme néo-libéral et anglo-saxon des études urbaines. Trois champs théoriques principaux peuvent être distingués dans cette littérature scientifique sur les jumelages.

Le premier champ se réfère au paradigme des relations internationales. Devant le constat de l'internationalisation croissante des villes et de leur compétition à l'échelle globale, les relations internationales étudient les jumelages entre les villes en termes de "diplomatie des villes" (local foreign policies en anglais) ou de paradiplomatie. La capacité des Etats à mener des relations extérieures est ainsi transposée aux villes dans le contexte de mondialisation (Vion, 2002 ; Viltard, 2008). A partir de la décennie 1980, le terme de coopération décentralisée est utilisé dans l'idée que la décentralisation de certaines compétences de l'Etat, comme la politique de coopération, peut être exercée par les villes. Cette littérature s'attache à une vision classique en termes d'analyse descendante ou ascendante (top-down et bottom-up) de la construction des liens de coopération, alors que de notre côté, nous nous appuyons sur l'idée que les jumelages de villes sont des liens faibles, ténus, plutôt faiblement institutionnalisés.

Le deuxième champ de recherche est partagé avec la géographie et porte sur l'internationalisation des villes et plus particulièrement sur le jeu d'échelle du local au global. La globalisation entraîne des effets de "glocalisation" (contraction de globalisation et local) qui consiste à penser l'action locale à l'échelle globale, le géographe britannique Eric Swyngedouw en est un des principaux théoriciens (Swyngedouw, 2004). Dans ce champ, les auteurs pensent les jumelages comme moyen pour les villes de s'insérer dans des niveaux supérieurs de la hiérarchie mondiale des villes (le rescaling ou la politique des échelles) et restent ainsi dans le paradigme de la compétition entre les villes (Clarke, 2009; Cremer, De Bruin et Dupuis, 2001). Les jumelages des villes sont l'expression d'une histoire unique de liens entre des cités, ils alimentent aussi la réflexion sur la gouvernance urbaine et le transferts de bonne pratiques entre les villes du Nord et celles du Sud (Bontenbal et van Lindert, 2009). Ils sont alors conçus comme des alliances stratégiques mises en place par les villes. Une telle approche, à rebours du premier champ théorique exposé, fait alors abstraction des 
niveaux intermédiaires entre le local et le global, tel l'État, pour comprendre la dynamique des jumelages. Or, dans un espace comme celui de la mer Noire traversé d'enjeux géopolitiques de premier ordre, une telle posture est difficilement tenable.

Enfin, un troisième champ de recherche se développe. Il s'appuie sur une approche par le niveau local pour comprendre l'intérêt des jumelages comme forme de coopération territoriale. Les jumelages sont alors conçus comme des instruments d'apprentissage et de connaissance mutuelle dans la durée, autour de valeurs ou d'expériences partagées (Zelinsky, 1991; Baldersheim, Bucek et Swianiewicz, 2002). L'internationalisation est étudiée du côté des pratiques comme l'hospitalité (Jayne, Hubbard et Bell, 2013), l'amitié et la bienveillance (Clarke, 2011). Le jumelage produit alors une proximité sociale entre les deux villes. De telles analyses, qui se rapprochent du champ émergent de la géographie des émotions, nécessitent une connaissance intime des histoires et des modalités de jumelages; elles rendent toutefois difficile la comparaison. Dans le cadre de cette étude, d'une part, nous n'avons pas pu mener d'enquêtes de terrain et nous privilégions, d'autre part, un regard multiscalaire qui fait s'articuler ville, pays, région mer Noire et échelle globale.

11 Pour cet article, nous considérons que les jumelages de villes sont une modalité de coopération territoriale (Bussi, 2009). De nombreuses études géographiques ont analysé la coopération territoriale transfrontalière et les eurorégions comme formes de coopération territoriale entre l'Europe et ses voisins, en fournir les jalons bibliographiques excède le champ de cet article. La place des villes dans les dynamiques mondiales justifie pleinement que leurs formes de coopération territoriale soient étudiées: la construction de réseaux internationaux de et les jumelages sont des actions peu visibles. Les jumelages sont des liens faiblement institutionnalisés qui naissent de simples connaissances, ils n'ont pas de forte implication financière. Ces liens faibles peuvent devenir forts quand ils permettent aux villes de pénétrer dans d'autres réseaux de villes et de tisser des liens avec d'autres niveaux de la hiérarchie urbaine, des premiers travaux ont exploré cette piste (Bucher et Grillon, 2008; Escach et Vaudor, 2014 ; Escach, 2015).

12 Les jumelages de villes sont nés de la Guerre froide : pendant que les Etats s'opposaient, les villes construisaient des coopérations (Chombard-Gaudin, 1992 ; Claeysen, 1999). On est alors tributaire de la bibliographie occidentale, alors qu'une exploration de la bibliographie en russe par exemple permettrait de comprendre la dynamique des jumelages, très active dans le bloc de l'Est, et dont le caractère politisé voire formel autour de l'amitié entre les peuples fournit une autre histoire (Zelinsky, 1991). La fin de la Guerre froide et l'émergence d'un monde multipolaire placent la mer Noire dans de nouveaux enjeux de confrontations et de coopérations (Kuşku-Sönmez, 2014). Les jumelages des villes de la mer Noire restent cependant un laboratoire privilégié pour étudier ces nouvelles dynamiques de coopération.

13 Par jumelage de villes, nous retenons la définition simple et commode du CCRE (Conseil des communes et régions d'Europe): «Les jumelages, c'est la rencontre de deux communes qui entendent proclamer qu'elles s'associent pour agir dans une perspective européenne, pour confronter leurs problèmes et pour développer entre elles des liens d'amitié de plus en plus étroits » (définition attribuée à Jean Bareth, président du CCRE vers 1951). Certes cette définition est européano-centrée et ancienne, mais elle est toujours valide. Elle permet de saisir la motivation des jumelages: partager ses difficultés, construire la paix par l'amitié et développer ses contacts. Dans la région de 
la mer Noire, cette définition garde tout son sens. Soixante ans après, une autre définition est tout aussi valable : « le jumelage est l'un des points de départ utilisé par les villes pour aspirer à une image distincte, visible et favorable et, en ce sens, il est partie prenante de leurs politiques de marketing urbain et d'image de marque dans un contexte de régionalisation transnationale d'intensité croissante" (Joenniemi et Sergunin, 2011, p. 231, nous traduisons). Située dans le paradigme de la ville néolibérale, actrice de son développement, cette définition montre l'impact de la mondialisation et de la compétition entre les villes dans la signification des jumelages. Les deux définitions retenues ne sont pas antithétiques, elles correspondent à des choix de stratégies urbaines.

\section{B. Questions et Méthodes d'analyse des jumelages de villes}

14 L'interrogation sur les jumelages des villes de la mer Noire s'inscrit dans un double mouvement. D'un côté, la vision géopolitique dominante des rives de la mer Noire est celle d'une espace fragmenté. D'un autre côté, l'accélération de l'ouverture internationale par les flux internes (mondialisation) et par les accords de coopération entre les pays bordiers montre une importante recomposition territoriale. L'étude des jumelages de villes concerne la question de l'intégration régionale au travers des liens de coopération. Nous avons emprunté la méthodologie au programme européen ESPON TERCO ${ }^{2}$ qui a étudié tous les jumelages des villes de l'Union européenne. Mais d'autres questions se sont posées pour le cas spécifique de la mer Noire.

15 La première question a porté sur les limites des pays bordiers de la mer Noire. Six pays sont côtiers de la mer Noire mais compte tenu de leur extension spatiale (pour la Russie et la Turquie tout particulièrement), il fallait trouver une limite pour les régions littorales. La définition d'Eurostat, l'institut statistique officiel de l'Union européenne, a été retenue car cette définition administrative est facilement transposable aux pays hors Union européenne. Elle concerne "les régions statistiques définies au niveau NUTS3 possédant plus de la moitié de leur population à moins de $50 \mathrm{~km}$ de la mer ». Le niveau NUTS3 correspond aux régions (oblast) en Bulgarie, aux départements roumains (judet), aux provinces turques (il) pour ces pays qui ont adopté la nomenclature européenne. En Russie, les kraj ont été retenus, en Ukraine les régions (oblast) et, en Géorgie, les républiques autonomes et les régions (mkhare). Les régions de Donetsk et Zaporižija ont été ajoutées en raison de leur lien avec les ports de Mariupol et Berdiansk.

16 La deuxième question portait sur la définition de la ville. Chaque pays a sa propre délimitation de la ville. Si l'on dresse un tableau comparatif en fonction des principaux indicateurs internationaux retenus dans la définition des villes, la diversité des situations exprime l'histoire de chaque pays. 


\begin{tabular}{|l|c|c|l|c|}
\hline Pays & Statut politique & Seuil de population & $\begin{array}{l}\text { Morphologie } \\
\text { urbaine }\end{array}$ & $\begin{array}{l}\text { Fonctions } \\
\text { économiques }\end{array}$ \\
\hline BULGARIE & $\mathrm{X}$ & & & \\
\hline ROUMANIE & $\mathrm{X}$ & & $\mathrm{X}$ \\
\hline UKRAINE & $\mathrm{X}$ & $\mathrm{X}$ & $\mathrm{X}$ \\
\hline RUSSIE & $\mathrm{X}$ & $\mathrm{X}$ & $\mathrm{X}$ \\
\hline GEORGIE & $\mathrm{X}$ & $\mathrm{X}$ & \\
\hline TURQUIE & $\mathrm{X}$ & $\mathrm{X}$ & & \\
\hline
\end{tabular}

Source : UNSTAT, demographic yearbook, table 6, 2005

statut politique : il est donné par les autorités politiques et il est complété par un critère démographique et d'un critère fonctionnel (prédominance de l'agriculture et part de l'emploi non-agricole dans les activités). Ce statut politique peut être décliné en plusieurs types selon la hiérarchie administrative des chefs-lieux. La Bulgarie et la Roumanie proposent une définition de la ville uniquement liée au statut politique, sans critère démographique ou fonctionnel. En Roumanie, plusieurs statuts sont distingués, ils conditionnent le montant des aides budgétaires. Enfin, en Turquie, la définition de la ville s'appuie sur le statut politique et un seuil de population qui varie selon le rang dans la hiérarchie administrative des chefs-lieux. Pour cette étude, la définition de la ville comme unité politique représentée le plus souvent par un maire et un conseil municipal nous intéresse car elle conditionne la capacité de la ville à pouvoir mener une action extérieure par un lien de jumelage. Dans ce cas, le statut politique de ville est important. On remarque d'ailleurs que pour tous ces pays, aucun critère morphologique, comme la continuité du bâti par exemple, n'est retenu.

En outre, nous avons adopté un seuil démographique significatif de la taille d'une ville afin de retenir les villes susceptibles d'avoir des jumelages et d'éliminer les trop petites villes de la base de données. Les études internationales s'accordent à retenir le seuil de 10000 habitants: c'est le seuil retenu par la base de données e-geopolis ${ }^{3}$ et la comparaison des villes du monde sur le temps long (Moriconi-Ebrard, 1994). L'adoption d'un tel seuil permet de s'affranchir de la question difficile de la différence entre espace urbain et espace rural pour tous les pays étudiés.

Une troisième question émerge alors, elle concerne le recensement des jumelages par ville. Les jumelages d'une ville avec une entité administrative d'un autre niveau (région, département etc) et les jumelages des villes à l'intérieur d'un même pays ont été éliminés. Suivant la méthode du programme ESPON TERCO, l'étude recense sur le site Internet wikipedia de chaque ville de la mer Noire les villes avec lesquelles cette ville déclare un jumelage international. Du point de vue de la comparaison entre pays, le site Internet en anglais de chaque ville offre le même modèle de tableau de jumelages. Ce tableau indique le nom de la ville jumelée, le pays et souvent la date du jumelage. En revanche, l'analyse perd en précision et en qualité de l'information: les renseignements sont très disparates d'une ville à l'autre et ils ne permettent pas de savoir si ce jumelage est actif ou inactif. 
Cette première liste de jumelages a donc été systématiquement croisée avec (1) la liste des jumelages urbains dressée pour chaque pays sur le site Internet wikipedia, (2) le site Internet wikipedia de la ville dans la langue nationale (même en géorgien, le tableau des jumelages s'identifie facilement sur la page web), (3) les sites Internet des villes concernées et leur page relations internationales, (4) les bases de données nationales quand elles sont disponibles (Bulgarie et Turquie notamment), (5) enfin, les bases de données internationales qui recensent pour l'Union européenne notamment les jumelages (base de données du Conseil des communes et régions d'Europe ${ }^{4}$ ). Un tel travail a pris plusieurs mois, il nécessiterait une collaboration plus étroite avec des collègues des pays concernés pour pouvoir vérifier et compléter. Ainsi, cet article présente une analyse exploratoire fondée sur la liste des lieux et des dates de jumelages complétée de la population des villes (vers 2010, selon les données de recensement disponibles auprès des instituts statistiques nationaux).

\section{Les jumelages des villes de la mer Noire : hypothèses et résultats}

La base de données comporte ainsi 99 villes de plus de 10000 habitants : la plus petite Büyükyoncalı compte 10072 habitants, et la plus grande, Istanbul 13 millions d'habitants, elles sont toutes deux en Turquie. Hormis Istanbul, trois villes sont millionnaires (Donetsk, Odessa et Rostov-sur-le-Don) et 4 autres ont plus de 500000 habitants (Mykolajv, Gebze, Krasnodar et Zaporižija). La base de données comptabilise 599 jumelages de villes avec 73 pays différents du monde entier. Elle couvre la période de 1944 à 2013, 76\% des jumelages informent sur la date du partenariat. Pour exploiter ces données, plusieurs hypothèses ont été formulées.

Tableau 2 : Présentation générale de la base de données sur les jumelages

\begin{tabular}{|l|l|l|l|l|l|l|l|}
\hline Pays & $\begin{array}{l}\text { Population } \\
\text { urbaine } \\
\text { concernée }\end{array}$ & $\begin{array}{l}\text { Nombre } \\
\text { de villes } \\
\text { avec } \\
\text { jumelages }\end{array}$ & $\begin{array}{l}\text { Nombre } \\
\text { de } \\
\text { jumelages }\end{array}$ & $\begin{array}{l}\text { Nombre } \\
\text { de pays } \\
\text { de } \\
\text { jumelages }\end{array}$ & $\begin{array}{l}\text { Nombre } \\
\text { moyen de } \\
\text { jumelages } \\
\text { par ville }\end{array}$ & $\begin{array}{l}\text { Nombre } \\
\text { moyen } \\
\text { d'habitants } \\
\text { par } \\
\text { jumelage }\end{array}$ & $\begin{array}{l}\text { Nombre } \\
\text { moyen de } \\
\text { jumelages } \\
\text { pour } \\
\text { 100 000 hab. }\end{array}$ \\
\hline Bulgarie & 695453 & 9 & 67 & 29 & 7,4 & 10380 & 9,6 \\
\hline Géorgie & 414545 & 5 & 41 & 13 & 8,2 & 10111 & 9,9 \\
\hline Roumanie & 530298 & 6 & 46 & 23 & 7,7 & 11528 & 8,7 \\
\hline Russie & 3959364 & 16 & 86 & 30 & 5,4 & 46039 & 2,2 \\
\hline Turquie & 17022454 & 39 & 181 & 60 & 4,6 & 94047 & 1,1 \\
\hline Ukraine & 6735837 & 24 & 178 & 39 & 7,4 & 37842 & 2,6 \\
\hline TOTAL & 29357951 & 99 & 599 & 80 & 6,1 & 49012 & 2,0 \\
\hline
\end{tabular}

Source : base de données des jumelages ESPON ITAN

\section{A. Hypothèse 1 : le nombre de jumelages est-il proportionnel à la population de la ville?}

Les études urbaines ont montré que la loi rang-taille permettait d'expliquer la place des villes dans la hiérarchie urbaine selon leur population. De la même façon, peut-on conclure que plus une ville est peuplée plus elle contracte d'accords de jumelages? 
Istanbul, ville d'importance mondiale est dix fois plus peuplée que Rostov-sur-le-Don, ville qui arrive en seconde position selon le rang de population. Istanbul cumule ainsi $10 \%$ des jumelages au total; elle a donc été exclue dans un premier temps de l'analyse.

Le modèle de régression linéaire (figure 1) sans Istanbul montre qu'un peu moins de $40 \%$ des jumelages peut s'expliquer par la relation proportionnelle entre le nombre de jumelages et la taille de la ville $\left(\mathrm{R}^{2}=0.3781\right)$. Parmi les villes de plus de 500000 habitants, seule Donetsk répond au modèle. Odessa se caractérise par un nombre de jumelages bien supérieur au modèle attendu en fonction de sa population : elle totalise 37 jumelages soit un tiers de jumelages de plus que Donetsk pour une population légèrement supérieure. Les autres villes situées en Russie, en Ukraine ou en Turquie (Rostov-sur-le-Don, Mykolajv, Gebze, Krasnodar et Zaporižija) ont un nombre de jumelages inférieur au modèle attendu. C'est le signe d'une internationalisation inachevée au regard de la taille de ces villes.

Figure 1 : Relation entre la population et le nombre de jumelages des villes de la mer Noire

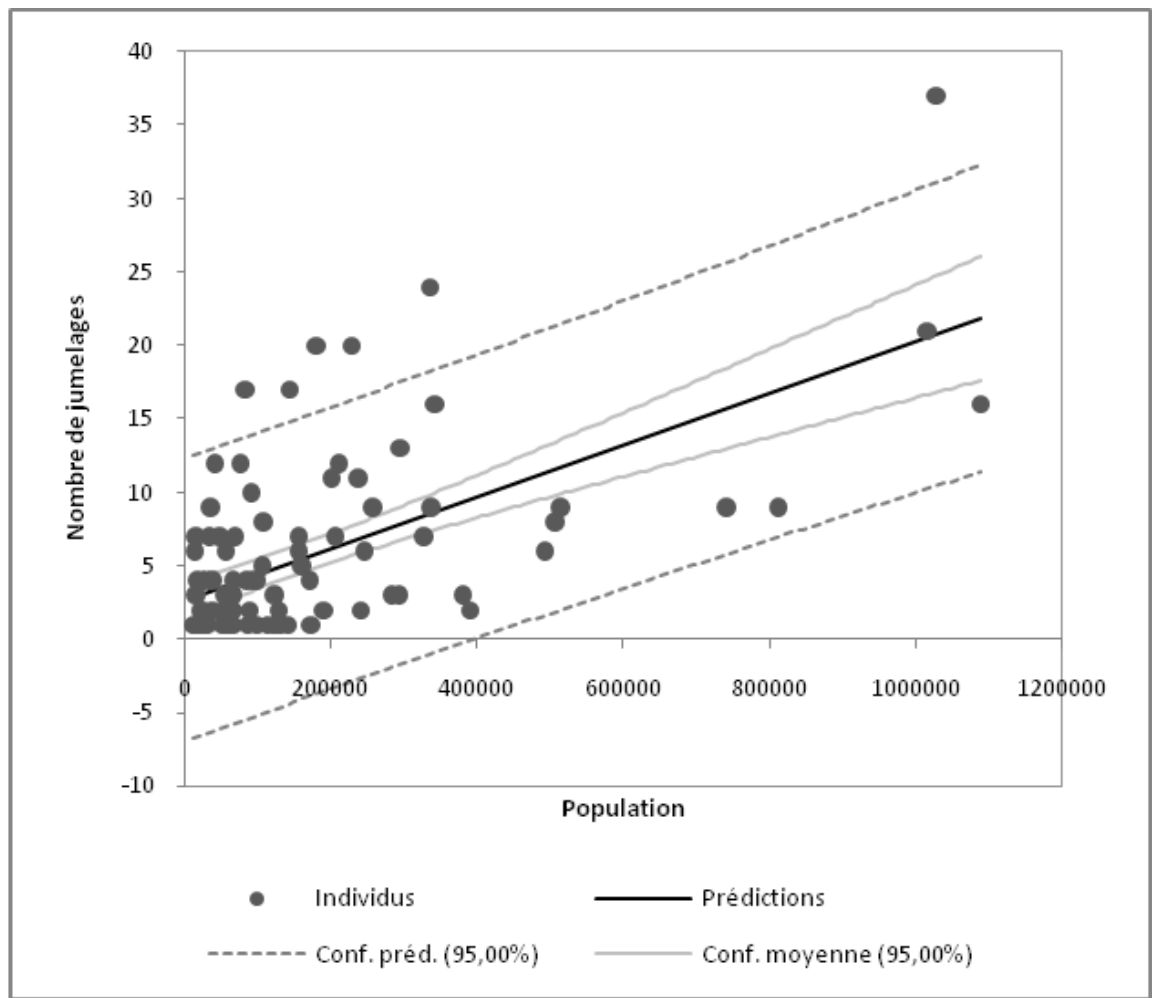

Source : base de données des jumelages ESPON ITAN

\section{B. Hypothèse 2 : quels sont les effets des recompositions post- socialistes?}

Interroger l'ouverture internationale des villes de la mer Noire au travers de leurs jumelages nécessite de questionner le changement politique majeur de 1989 pour la Bulgarie et la Roumanie et de 1991 pour les pays issus de l'URSS. Le tournant de la décennie 1990 marque l'émergence d'un monde multipolaire où la mer Noire retrouve sa position de carrefour entre l'Asie, l'Europe et le Proche-Orient. On en voit les effets sur le graphique du nombre de jumelages dans le temps (figure 2). La décennie 1990 
marque un renouveau des jumelages internationaux des villes, cela se fait même sentir dés la fin des années 1980. La décennie 2000 marque un réel développement des jumelages et une ouverture internationale.

Figure 2 : Nombre de jumelages des villes de la mer Noire conclus entre 1944 et 2013

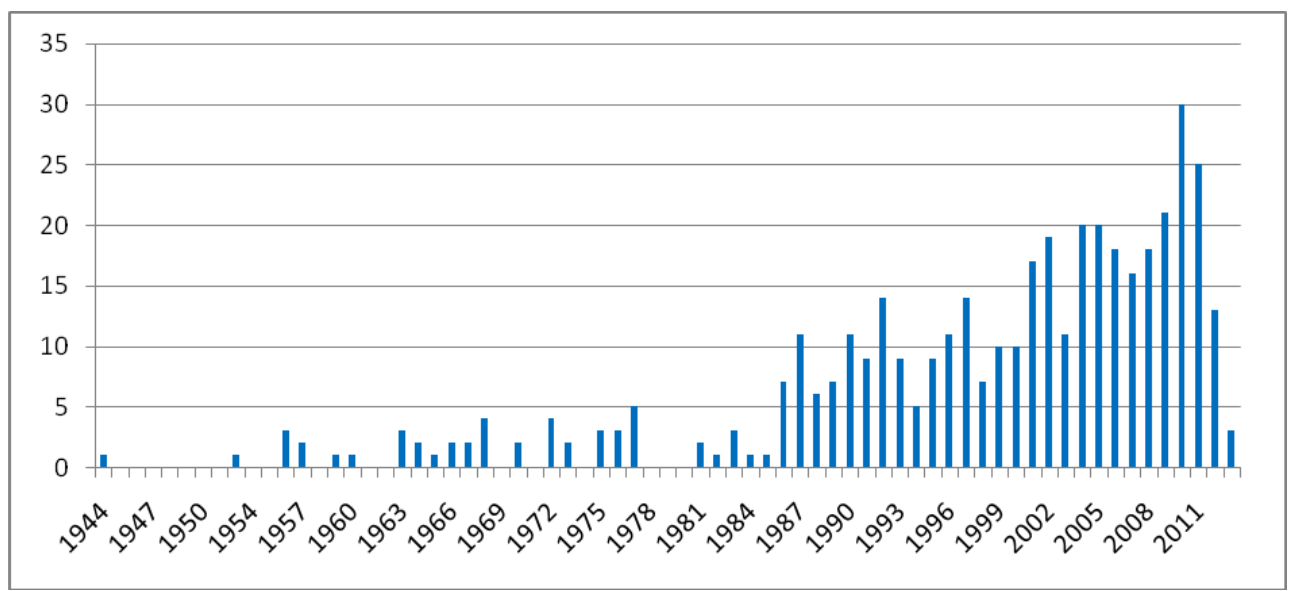

Source : base de données des jumelages ESPON ITAN

Les recompositions géopolitiques se traduisent dans la géographie des jumelages (figure 3). Avant 1990, les jumelages des villes de la mer Noire étaient tournés vers l'Europe occidentale et des pays très investis dans le développement des jumelages (la France et l'Allemagne par exemple). Le tournant de 1990 montre que les nouveaux jumelages ont un rayon d'action régionale avec l'ouverture immédiate aux jumelages entre pays voisins. Il montre aussi le retour de l'intérêt géopolitique pour le bassin de la mer Noire : les Etats-Unis, la Russie, la Turquie et la Chine se positionnent tout de suite parmi les principaux pays de jumelages urbains. En première position, l'Allemagne réunifiée contribue au développement de la coopération territoriale entre ancien blocs de l'Est et de l'Ouest. L'augmentation du nombre de jumelages accompagne une grande diversité géographique des jumelages dans la décennie 2000. Les nouveaux jumelages entre pays bordiers de la mer Noire dominent par leur nombre (Russie, Bulgarie, Turquie), mais les ouvertures sont méditerranéennes (Italie, Grèce, Maroc, Israël) ou largement internationales (Chine, Mexique, Iran). Dans ce dernier cas, les grandes villes portuaires concluent un jumelage avec une ville-port de la mer Noire. 
Figure 3 : Répartition chronologique et géographique des jumelages des villes de la Mer noire

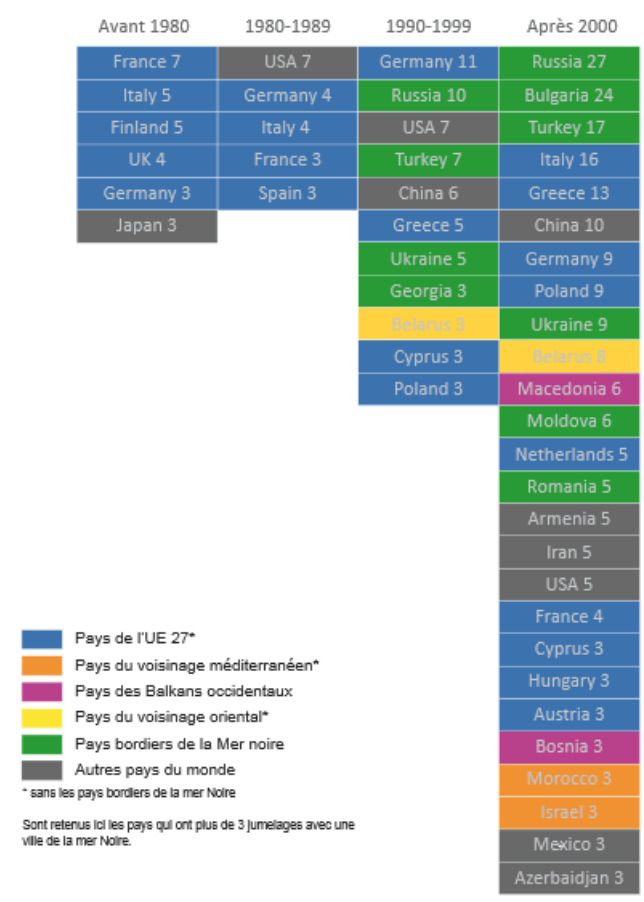

\section{Hypothèse 3 : La proximité spatiale joue-t-elle?}

Dans les stratégies urbaines pour lier des jumelages, on peut penser que la proximité spatiale joue. Selon cette hypothèse, les réseaux de villes se construisent sur de l'interconnaissance dans un premier temps, afin d'augmenter le cercle de contacts internationaux dans un second temps. L'analyse dans le détail des jumelages internationaux des villes de la mer Noire nuance cette hypothèse.

Toutes périodes cumulées, la Russie et la Turquie apparaissent parmi les trois premiers pays destinataires des jumelages (respectivement 63 et 36 jumelages accueillis). Cette place s'explique par deux faits: leur poids régional en terme géopolitique et économique d'une part, leur poids démographique en termes de population urbaine dans la région de la mer Noire d'autre part (tableau 1). La deuxième place de la Bulgarie (43 jumelages) est plus étonnante, elle s'explique en partie par le fort nombre de jumelages entre les villes turques et les villes bulgares à fort peuplement turc. Un groupe de quatre pays (autour de 30 jumelages accueillis) apparaît ensuite : il regroupe l'Ukraine, l'Italie, l'Allemagne et la Grèce. Les trois derniers sont des pays européens aux fortes cultures urbaines et de jumelages. La présence de la Grèce renvoie à son importance historique dans la mer Noire pendant l'Antiquité avec le système des comptoirs. Le poids démographique urbain explique la position de l'Ukraine. Enfin, en $8^{e}$ et $9^{e}$ place, les Etats-Unis (26 jumelages) et la Chine (21 jumelages) montrent l'ouverture internationale à d'autres horizons des villes de la mer Noire: les grands ports chinois et américains sont les principaux destinataires de ces jumelages.

Au total, les jumelages des villes de la mer Noire se ventilent de façon parfaitement égale entre les six pays bordiers de la mer Noire (207 jumelages soit $34 \%$ ) et les pays 
européens (UE-25, Suisse et Norvège), les autres pays du monde accueillent 185 jumelages (soit $32 \%$ ). On voit ainsi que la mer Noire est traversée d'un double mouvement: celui d'une régionalisation interne qui passe par le renforcement des coopérations entre les villes du pourtour de la mer Noire et celui d'une ouverture internationale vers les autres continents. Le tropisme européen peut s'expliquer par le fait que deux pays, la Bulgarie et la Roumanie, sont membres de l'UE depuis 2007 et que la Turquie est pays candidat. Ces rapprochements ont joué, ils s'ajoutent au fait que les pays européens sont aussi très actifs dans les jumelages. L'Union européenne soutient ainsi politiquement et financièrement ces initiatives, au travers notamment de son programme «l'Europe pour les citoyens ».

Figure 4 : Appartenance géographique des pays placés en première position dans les jumelages

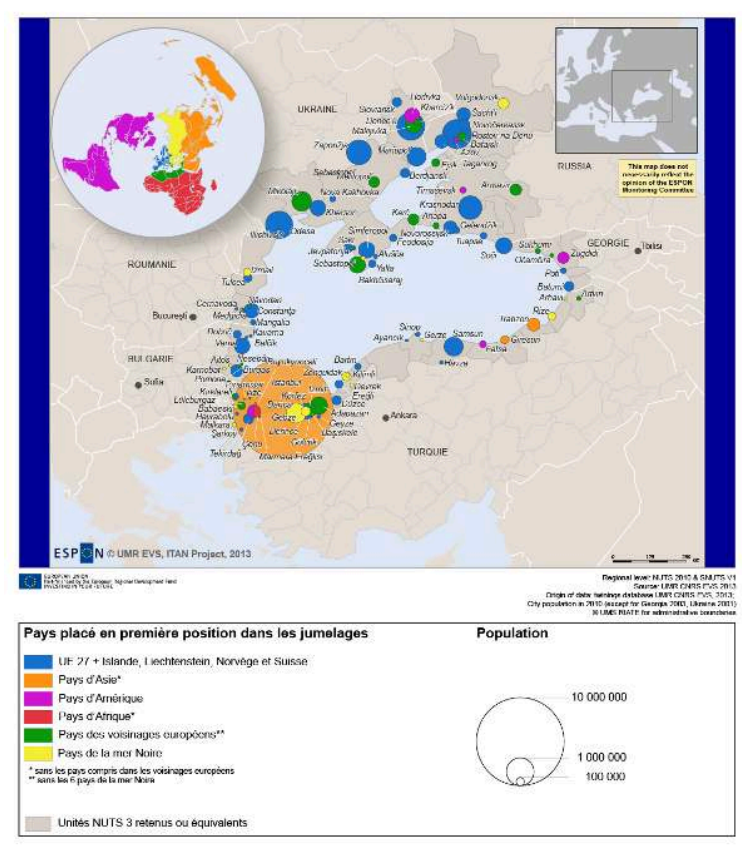

Source : ESPON ITAN

La distribution géographique des jumelages, selon l'appartenance continentale des villes jumelles (figure 4), montre que les villes dont les jumelages sont préférentiellement tournés vers les autres rives de la mer Noire sont peu nombreuses (15 unités). La Bulgarie et la Roumanie, sans surprise, sont davantage tournées vers l'Europe. Les villes ukrainiennes, à l'exception d'Horlivka (sur deux jumelages, deux sont avec des villes américaines), se tournent préférentiellement vers les voisinages immédiats de la mer Noire (Balkans, Caucase, Biélorussie, rive sud de la Méditerranée) ou vers l'Europe. Il en est de même pour la Russie. En Géorgie, les jumelages se partagent entre l'Europe et les voisinages immédiats de la mer Noire, l'unique jumelage international de Zugdidi avec Columbus aux Etats-Unis ne peut pas être considéré comme significatif. Enfin, la Turquie offre un panorama très coloré des orientations géographiques de ses jumelages urbains. Istanbul regroupe à elle seule $10 \%$ des jumelages, et le tiers de ceux-ci sont tournés vers l'Asie et ses principaux ports chinois, 
malaisiens, coréens et japonais. L'internationalisation de cette ville portuaire, carrefour mondial, est évidente. Les autres principaux ports turcs de la mer Noire sont tournés vers l'Asie : l'Iran et la Chine pour Trabzon, l'Iran et le Japon pour Giresun. Samsun est de peu tournée vers les villes européennes (Kiel en Allemagne, Kalmar en Suède et Iskele à Chypre) mais garde un jumelage avec Novorossijsk avec laquelle elle est reliée par gazoduc.

Enfin, un dernier focus sur les principales villes portuaires montre des degrés d'ouverture internationale divers. Après Istanbul, Odessa est la deuxième ville qui présente le plus de jumelages (37) alors qu'elle est dix fois moins peuplée que la métropole turque : sur 37 jumelages recensés, 28 sont réalisés avec des grandes villes portuaires mondiales de tous les continents (Vancouver, Valparaiso, Qingdao, Alexandrie, Kolkota, Haifa, Yokohama etc) et parfois de longue date (depuis 1957 pour Oulu en Finlande et Liverpool en Grande-Bretagne). Dans le courant de la décennie 1990, la ville a développé des jumelages avec des capitales d'Etat (Istanbul, Minsk, Moscou, Nicosie, Vienne et Erevan) ce qu'elle ne pouvait pas faire à l'époque soviétique où le respect des hiérarchies de chefs-lieux devait être respecté dans les jumelages. Une autre ville millionnaire par la population, Rostov-sur-le-Don, présente un profil de jumelage très différent: la ville n'a noué que 16 jumelages (dont sept avec des villes anciennement soviétiques) et seulement cinq avec des villes portuaires (Antalya en Turquie, Glasgow en Grande-Bretagne, Mobile aux Etats-Unis, Odessa en Ukraine et Volos en Grèce). Cette faible internationalisation pour une ville de cette ampleur peut s'expliquer par le rôle de Rostov comme plateforme intérieure du système des cinq mers en Russie.

\section{Conclusion}

L'étude des jumelages internationaux des villes de la mer Noire présente plusieurs points d'intérêt. La dynamique temporelle montre une accélération des jumelages à partir des années 2000 : ces formes de coopération contribuent à l'internationalisation des villes. Les jumelages de villes s'inscrivent aussi dans le mouvement des nombreuses initiatives de coopération, dans une mer Noire au cœur des nouvelles recompositions géopolitiques mondiales.

La dynamique spatiale inscrit les jumelages dans le mouvement de régionalisation propre à la mondialisation. Du côté de la régionalisation, les jumelages sont noués pour un tiers d'entre eux entre les pays bordiers de la mer Noire. Les édiles urbains ont pris conscience de l'intérêt de coopérer entre villes pour construire et renforcer un réseau de relations urbaines. L'élargissement des contacts par jumelage inscrit ces villes dans la mondialisation : l'Asie et l'Amérique sont bien représentées notamment au travers de jumelages avec leurs principales villes portuaires. L'Afrique, comme dans d'autres secteurs de la mondialisation est faiblement représentée et se limite à son rivage méditerranéen.

Il reste cependant beaucoup de pistes à explorer, elles tiennent aux limites de cette étude. La base de données construite ne donne aucune information sur le degré d'activité du jumelage ni sur le ou les thèmes qui ont conduit au rapprochement entre deux villes. Ces deux informations permettraient d'affiner l'analyse. Enfin, une collaboration avec des chercheurs des pays de la mer Noire permettrait de vérifier par des enquêtes de terrain les informations; les données sur les motivations de telles 
initiatives, les formes et l'histoire de ces liens seraient l'occasion de les replacer dans les évolutions géopolitiques, sociales et économiques de ces pays. Enfin, l'échelle locale des jumelages de villes contribue à la dimension transnationale de l'internationalisation, il faudrait maintenant comprendre si cela se fait avec l'aval ou en contournant les Etats pour tester l'hypothèse du glocal.

\section{BIBLIOGRAPHY}

Baldersheim H., Bucek J., Swianiewicz P., 2002, « Mayors Learning across Borders: The International Networks of Municipalities in East-Central Europe », Regional \& Federal Studies, vol. $12, \mathrm{n}^{\circ} 1, \mathrm{p} .126-137$.

Beckouche P., 2013, « Atlas des intégrations régionales. Euro-Méditerranée, Asie orientale, Amérique du Nord », IPEMED, consulté le 28 mai 2015, http://www.ipemed.coop/fr/publicationsr17/etudes-analyses-c108/atlas-des-integrations-regionales-euro-mediterranee-asie-orientaleamerique-du-nord-a2139.html

Bontenbal M., van Lindert P., 2009, « Transnational city-to-city cooperation: Issues arising from theory and practice », Habitat International, vol. 33, n², p. 131-133.

Boulineau E., 2016, La coopération territoriale enter l'Union européenne et ses voisinages orientaux et balkaniques. Contribution à une géographie politique de l'européanisation. volume 1: position et projet scientifiques, Habilitation à Diriger des Recherches, ENS de Lyon, 207 p.

Bucher K., Grillon N., 2008, « Les jumelages stratégiques au service de la compétition entre les villes ", Revue Géographique de l'Est, vol. 48, n³/4, mis en ligne le 02 mars 2010, consulté le 3 décembre 2014. URL : http://rge.revues.org/1729

Bussi M., 2009, Un monde en recomposition : géographie des coopérations territoriales, Mont-SaintAignan, Publications des Universités de Rouen et du Havre, 316 p.

Chombard-Gaudin c., 1992, «Pour une histoire des villes et communes jumelées », Vingtième Siècle. Revue d'histoire, vol.35, n¹, p. 60-66.

Claeysen C., 1999, «Les jumelages et l'Europe », Annuaire des collectivités locales, vol. 19, n¹, p. $125-138$.

Clarke N., 2009, « In what sense 'spaces of neoliberalism'? The new localism, the new politics of scale, and town twinning ", Political Geography, vol. 28, n8, p. 496-507.

Clarke N., 2011, « Globalising care? Town twinning in Britain since 1945 », Geoforum, vol. 42, n²1, p. $115-125$.

Cremer R.D., De Bruin A., Dupuis A., 2001, « International Sister-Cities: Bridging the Global-Local Divide ", American Journal of Economics and Sociology, vol. 60, n¹, p. 377-401.

Drevet J.-F., 2010, « Confrontation ou coopération dans le bassin de la mer noire ? ", Confluences Méditerranée, vol.74, n³, p. 119-132. 
Escach N., 2015, «Les réseaux de villes baltiques : la dimension spatiale de la recomposition des niveaux ", L'Information géographique, vol. 79, n³, p. 34-53.

Escach N., Vaudor L., 2014, « Réseaux de villes et processus de recomposition des niveaux : le cas des villes baltiques », Cybergeo : European Journal of Geography [En ligne], Politique, Culture, Représentations, document 679, mis en ligne le 05 juillet 2014, consulté le 06 novembre 2014. URL : http://cybergeo.revues.org/26336; DOI : 10.4000/cybergeo.26336

Jayne M., Hubbard P., Bell D., 2013, « Twin Cities: Territorial and Relational Geographies of "Worldly" Manchester ", Urban Studies, vol.50, n², p. 239-254.

Joenniemi P., Sergunin A., 2011, « When Two Aspire to Become One: City-Twinning in Northern Europe ", Journal of Borderlands Studies, vol. 26, n², p. 231-242.

Kuşku-Sönmez E., 2014, « Regional Cooperation in the Black Sea Basin: what Role for City Diplomacy?», Southeast European and Black Sea Studies, vol. 14, n², p. 489-507.

Moriconi-Ebrard F., 1994, Geopolis : pour comparer les villes du monde, Paris, Anthropos , 246 p. Serebrian 0., 2010, Autour de la mer Noire : géopolitique de l'espace pontique, Perpignan, Artège Sciences humaines, $256 \mathrm{p}$.

Sieca-Kozlowski E., Toumarkine A., 2000, Géopolitique de la mer Noire: Turquie et pays de l'ex-URSS, Paris, Karthala, 222 p.

Swyngedouw E., 2004, "Globalisation or "Glocalisation"? Networks, Territories and Rescaling ", Cambridge Review of International Affairs, vol. 17, $n^{\circ} 1$, p. 25 - 48.

Viltard Y., 2008, «Conceptualiser la « diplomatie des villes » ", Revue française de science politique, vol.58, $\mathrm{n}^{\circ} 3$, p. $511-533$.

Vion A., 2002, «Europe from the Bottom up: Town Twinning in France during the Cold War », Contemporary European History, vol.11, n 4, p. 623 - 640.

Zelinsky w., 1991, « The Twinning of the World : Sister Cities in Geographic and Historical Perspective ", Annals of the Association of American Geographers, vol.81, n¹, p. 1-31.

\section{NOTES}

1. Programme de recherché ESPON ITAN (Integrated territorial analysis of the neighbourhood). Tous les résultats en anglais sont disponibles sur le site: http://www.espon.eu/main/ Menu_Projects/Menu_AppliedResearch/itan.html. Les propos tenus dans cet article n'engagent que leur auteur.

2. ESPON TERCO http://www.espon.eu/main/Menu_Projects/Menu_AppliedResearch/ terco.html.

3. http://e-geopolis.eu/?lang=en.

4. http://www.twinning.org. 


\section{ABSTRACTS}

Town-twinnings are still under-considered in research regarding their contribution to the urban strategies of internationalization. The paper focuses on the town-twinnings of the cities belonging to the Black sea states and aims at understanding how these local initiatives contribute to their integration into the globalization, in a context of an uncomplete regionalization of the Black sea space. The tension between the geopolitical fragmentation and the territorial cooperation through town-twinnings within and outside the Black sea space leads the demonstration in a space and time approach. The town-twinnings database was built in the framework of the ESPON ITAN European research project. It gathers the town-twinnings of the following countries: Bulgaria, Romania, Ukraine, Georgia, and Russia.

Les jumelages de villes constituent une entrée encore peu explorée pour comprendre les stratégies d'internationalisation des villes. L'étude des jumelages contractés par les villes des pays bordiers de la mer Noire présentée ici permet de regarder dans un espace en voie de structuration régionale comment ces actions locales contribuent à l'insertion des villes dans la mondialisation. La tension entre fragmentation géopolitique et coopération territoriale par les jumelages sert de fil conducteur à la compréhension dans le temps et dans l'espace des dynamiques des jumelages de villes autour de la mer Noire. Cette étude s'appuie sur une base de données constituée dans le cadre du programme de recherche ESPON ITAN. Elle recense les jumelages de villes des six pays suivants : Bulgarie, Roumanie, Ukraine, Russie, Géorgie, Turquie.

Die Städtepartnerschaften bilden einen noch wenig erforschten Ansatz, um die Internationalisierungsstrategien der Städte $\mathrm{zu}$ verstehen. Die hier vorgestellte Studie der Städtepartnerschaften mit Vertrag durch die Städte der an der Grenze zum Schwarzen Meer gelegenen Länder ermöglicht es, in einem sich auf dem Weg zur Strukturierung befindlichen Raum zu betrachten, wie diese lokalen Maßnahmen zur Integration der Städte in der Globalisierung beitragen. Die Spannung zwischen geopolitischer Fragmentierung und territorialer Zusammenarbeit durch Partnerschaften dient als Leitlinie für das Verständnis der zeitlichen und räumlichen Dynamiken der Städtepartnerschaften im Gebiet umdas Schwarze Meer. Diese Studie basiert auf einer im Rahmen des Forschungsprogramms ESPON ITAN erstellten Datenbank. Sie umfasst die Städtepartnerschaften der folgenden sechs Länder: Bulgarien, Rumänien, die Ukraine, Russland, Georgien, die Türkei.

\section{INDEX}

Schlüsselwörter: geopolitische Zersplitterung, Globalisierung, Internationalisierung, Regionalisierung, Schwarzes Meer, Städte, Städtepartnerschaften

Keywords: Black sea, cities, geopolitical fragmentation, globalization, internationalization, regionalization, Town-twinning

Mots-clés: fragmentation géopolitique, internationalisation, jumelages, mer Noire, mondialisation, régionalisation, villes 
AUTHOR

\section{EMMANUELLE BOULINEAU}

Maître de conférences HDR en géographie, Univ Lyon, CNRS, ENS de Lyon, EVS, UMR5600,

F-69007, France, emmanuelle.boulineau@ens-lyon.fr 